\title{
INFLUENCIA DEL TIEMPO Y TEMPERATURA DE FRITURA EN LA TEXTURA Y PERFIL LIPÍDICO EN CARNE DE CUY (Cavia porcellus) FRITA
}

\author{
INFLUENCE OF FRYING TIME AND TEMPERATURE ON TEXTURE AND \\ LIPID PROFILE IN GUINEA PIG MEAT (Cavys) FRIED
}

\author{
'Gina Genoveva Toro Rodríguez; ${ }^{2}$ Thomas Ancco Vizcarra; ${ }^{2}$ David Juan Ramos Huallpartupa
}

\begin{abstract}
RESUMEN
En la presente investigación se determinó la influencia del tiempo y la temperatura en la textura y perfil lipídico en carne de cuy (Cavia porcellus) frita. Se evaluó el tiempo ( 8,10 y 13 minutos) y la temperatura $\left(160,170\right.$ y $\left.180^{\circ} \mathrm{C}\right)$ de fritura en carcasas de Cavia porcellus deshuesadas provenientes del distrito de San Jerónimo - Andahuaylas, utilizándose aceite de soja y una freidora eléctrica con control de tiempo y temperatura. Para los análisis de textura se utilizó el equipo analizador de textura modelo TA-XT2 del Reino Unido y para el perfil lipídico el equipo cromatógrafo de gases. Se analizaron los parámetros texturales de elasticidad, cohesividad, masticabilidad, gomosidad y dureza a un nivel de confianza del $95 \%$, existiendo evidencia estadística para afirmar que la temperatura tiene un efecto sobre la elasticidad y la dureza, el tiempo y la temperatura tienen efecto sobre la cohesividad; sin embargo el tiempo y la temperatura no tienen un efecto sobre la cohesividad y la masticabilidad. En el perfil de ácido grasos las muestras evidencian la presencia de ácidos grasos entre ellos ácido linoléico, linolénico, eládico, palmítico, esteárico, oleico, heptadecanoico, mirístico y pentadecanoico respectivamente, la mayor cantidad presente es el ácido graso linoleico y el de menor cantidad el ácido pentadecanoico.
\end{abstract}

Palabras clave: Carne de cuy, textura y ácido graso.

\section{ABSTRACT}

During this investigation it was determined the influence of the effect of the time and temperature on the texture and fat components of fried cuy (Cavia porcellus) meet. It was evaluated different time frames ( 8,10 , and 13 minutes) and different temperature ( 160,170 and 180 ${ }^{\circ} \mathrm{C}$ ) to fry boneless cuy in carcasas of Cavia pocellus from the district of San Jeronimo- Andahuaylas using soy oil and electrical fryer with controlled time and temperature. For this texture analysis it was used an equipment that analysis the texture model TA-XT2 from Reino Unido and for the lipid profile a gas chromatographic equipment. It was analyzed the following parameters: elasticity, cohesiveness, chewable, gumminess, and hardiness to a $95 \%$ level with statistics evidence to state that the temperature has effect on the elasticity and hardness. The time and temperature have effect on the cohesiveness. However the time and temperature have no effect over the cohesiveness and chew ability. For the fatty acid profiles, the analysis show evidence of the presence of fatty acids which are linoleic acid, linoleic, eladic, palmitic, steric, oleic, heptadecanoic, miristic, and pentadecanoic. The mayor component is linoleic fatty acid and the smallest amount of pentadecanoic acid.

Keywords: Cuy meet, texture and fatty acids.

\section{INTRODUCCIÓN}

Se entiende por fritura al proceso que consiste en introducir un alimento en un baño de aceite o grasa caliente a temperaturas elevadas $\left(150-200^{\circ} \mathrm{C}\right)$, donde el aceite actúa de trasmisor del calor produciendo un calentamiento rápido y uniforme del producto (Yague, 2003). Se denomina fritura profunda a la sumersión total del alimento en el baño de fritura. Existen cambios y alteraciones al aumentar la temperatura, acelerándose todos los procesos químicos y enzimáti- cos, entre los que se evidencian la hidrolisis, oxidación, polimerización.

La textura es un atributo sensorial de importancia suprema para la preferencia de un producto frito y es un parámetro crítico en la calidad final del producto (Kita et al., 2007). Se espera que un producto frito de buena calidad tenga una corteza crujiente entre 1 y $2 \mathrm{~mm}$ como un indicador de frescura y alta calidad, y un centro cocido, húmedo y suave (Sanz et al., 2007). El análisis de perfil de textura, TPA por sus siglas en inglés, es una simulación de la masticación

\footnotetext{
Ingeniero Agroindustrial. Escuela Profesional de Ingeniería Agroindustrial de la Universidad Nacional José María Arguedas. Apurimac-Perú.

Magister Scientiae en Postcosecha y Marketing, Ingeniero Agroindustrial. Escuela Profesional de Ingeniería Agroindustrial de la Universidad Nacional José María Arguedas. Apurimac-Perú.
} 
Toro, G. et al. Influencia del tiempo y temperatura de fritura en la textura y perfil lipídico cn carne de cuy (cavia porcellus) frita.

de una muestra por medio de un equipo analizador de textura.

Consiste en comprimir un alimento del tamaño de un mordisco para imitar la acción de los dientes e incluye el análisis de diferentes características de textura de una muestra (Taiwo y Baik, 2007; Hleap y Velasco, 2010; TaveraQuiroz et al., 2012).

Cuando se fríe, el alimento se enriquece en grasa y éste enriquecimiento depende del contenido de grasa del alimento crudo. Según Perez (1997), el enriquecimiento lipídico supone un incremento en el valor energético del alimento y puede contribuir además al transporte de componentes liposolubles, tales como los ácidos grasos insaturados y posiblemente las vitaminas liposolubles. Del mismo modo, la grasa absorbida por el alimento procedente del medio de fritura puede contribuir a la obtención de un producto frito más equilibrado en ácidos grasos desde el punto de vista de la salud (Sánchez-Muniz et al., 1992). El presente estudio tiene la finalidad de determinar la influencia de la temperatura y el tiempo en la textura y perfil lipídico en la carne de cuy frita.

\section{MATERIALES Y MÉTODOS}

Lugar de ejecución: El trabajo de investigación se realizó en el Laboratorio de Análisis Físico-Químico de los Alimentos, Laboratorio de microbiología, Laboratorio de Investigación y en la Planta Piloto de Alimentos (TAPA) de la Facultad de Industrias Alimentarias de la Universidad Nacional Agraria La Molina.

\section{Materiales}

Materia prima: Carne de cuy deshuesada de la raza peruanita proveniente del distrito de Talavera, provincia de Andahuaylas y región Apurímac.

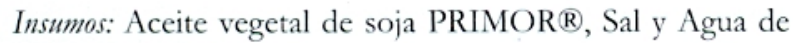

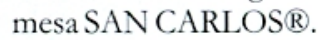

Materiales: Tablas de picar de de $50 \times 30 \mathrm{~cm}$, cuchillos de acero inoxidable, bol de acero inoxidable, papel toalla, papel absorbente, trinches de acero inoxidable y pinzas de acero inoxidable y guantes para soportar temperatura, bolsas de aluminio, bolsas ziflop, baguetas, beakers de $100 \mathrm{~mL}$ y 250 $\mathrm{ml}$, fiolas de $10 \mathrm{~mL}, 25 \mathrm{~mL}$ y $50 \mathrm{~mL}$, matraz de Erlenmeyer de $50 \mathrm{~mL}$ y $100 \mathrm{~mL}$, probeta de $250 \mathrm{~mL}$ y $500 \mathrm{~mL}$, bureta bombilla de succión y termómetro.

Equipos: Freidora eléctrica, marca Diana, modelo DN-2002. Determinador de actividad de agua AquaLab®, Analizador de Textura modelo'TA-X'T2, Stable Micro Systems del Reino, Balanza de precisión $(0,1 \mathrm{~g})$, Balanza analítica $(0,0001 \mathrm{~g})$, estufa, Incubadora, equipo soxhlet, Cromatógrafo de gases.

\section{Metodología de análisis}

Elaboración de carne de cuy frita: El diagrama de flujo de las operaciones a realizar, para la obtención de la carne de cuy frita, se observa en la figura 1.

La carne de cuy frita que se obtuvo fue de carcasas deshuesadas y cortadas por la mitad, fritas con aceite de soja en una relación cuy aceite de $1 / 6$ y sometidas a fritura utilizándose tres temperaturas $\left(160,170\right.$ y $\left.180^{\circ} \mathrm{C}\right)$ y tres tiempos $(8,10$ y 13 minutos) obteniéndose nueve tratamientos. (T1: $160^{\circ} \mathrm{C}$ a $8 \mathrm{~min}, \mathrm{~T} 2: 160^{\circ} \mathrm{Ca} 10 \mathrm{~min}, \mathrm{~T} 3: 160^{\circ} \mathrm{Ca} 13 \mathrm{~min}, \mathrm{~T} 4:$ $170^{\circ} \mathrm{Ca} 8 \mathrm{~min}, \mathrm{~T} 5: 170^{\circ} \mathrm{Ca} 10 \mathrm{~min}, \mathrm{~T} 6: 170^{\circ} \mathrm{Ca} 13 \mathrm{~min}, \mathrm{~T} 7$ : $180^{\circ} \mathrm{Ca} 8 \mathrm{~min}, \mathrm{~T} 8: 180^{\circ} \mathrm{Ca} 10 \mathrm{~min}$ y T9: $\left.180^{\circ} \mathrm{Ca} 13 \mathrm{~min}\right)$.

Controles: Se realizó un estudio de la influencia de la textura y perfil lipídico en la carne de cuy frita; realizándose análisis proximal, índice de peróxido, actividad de agua, textura (cohesividad, gomosidad, elasticidad, masticabilidad y dureza) y perfil lipídico. A continuación se explica el procedimiento de los análisis. Condiciones ambientales $\left(20^{\circ} \mathrm{C}\right.$ y humedad relativa $89 \%$ ).

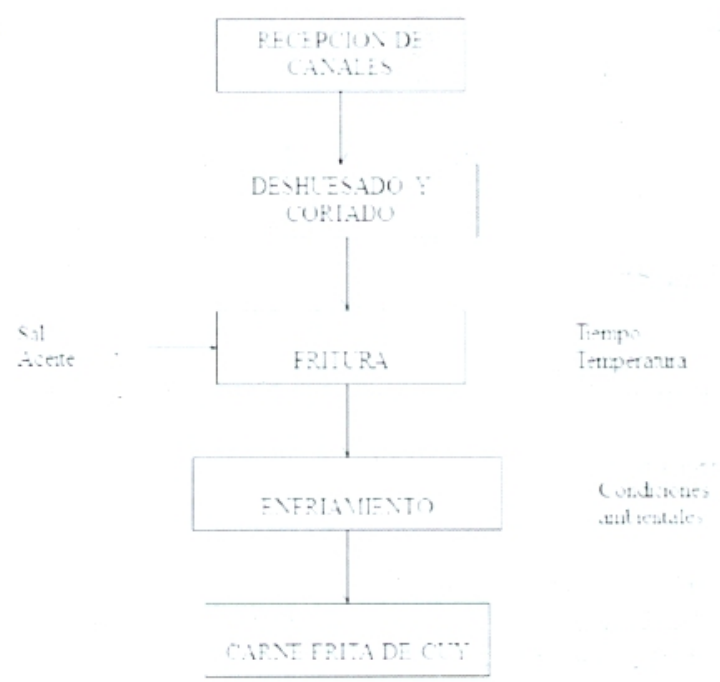

Figura 1. Elaboración de carne de cuy frita

Análisisproximal:

- Humedad: Método de la AOAC (2005). Método 934.06.

- Grasa: Método de la AOAC (2000). Método 9761.46

- Cenizas: Método de la AOAC (2005). Método 940.26

- Proteína: Método de la AOAC (2000). Método 981.10.

Indice de peróxido: Método AOAC (1990). Método 965.33.

Actividad de agua: Se utilizó un higrómetro de punto de rocío AquaLab. AOAC Oficial Method 32.005.

Textura: Las muestras para el análisis de perfil de textura (TPA) (Bourne, 1978) se obtendrán cortando cubos de $1 \mathrm{~cm}$ de cada lado paralelo a la dirección de la fibra muscular, y después se comprimirán a 75\% $(7,5 \mathrm{~mm})$ utilizando una velocidad de cruceta de $5 \mathrm{~mm} / \mathrm{s}$. determinándose los factores texturales de Elasticidad (Ad.), cohesividad (Ad) gomosidad $\left(\mathrm{Kg} \mathrm{m} \mathrm{s}^{-2}\right)$, masticabilidad $(\mathrm{Kg})$ y la dureza $\left(\mathrm{Kg} \mathrm{m} \mathrm{s}^{-2}\right)$ en un analizador de textura modeloTA-XT2 (Stable Micro Systems, Reino Unido).

Perfil de ácido graso: Método Cromatografía de Gases (AOAC, 996.06). 
Toro, G. et al. Influencia del tiempo y temperatura de fritura en la textura y perfil lipídico en carne de cuy (cavia porcellus) frita.

\section{Análisis estadístico}

En los resultados fisicoquímicos en el producto terminado, se realizó una prueba de comparación simple con la finalidad de determinar si existen diferencias significativas entre las muestras y el control (Gutiérrez y de la Vara, 2003). Todos los análisis estadísticos se desarrollaron para una probabilidad del 95\% de confianza, con el programa estadístico Statgraphics Centurion.

\section{RESULTADOS Y DISCUSIONES}

\section{Análisis proximal:}

En la tabla 1 se muestra el análisis proximal realizados a los diferentes tratamientos.

Tabla 1. Valores de análisis proximal en porcentaje

\begin{tabular}{ccccc}
\hline Tratamiento & Humedad \% & Proteina $\%$ & Grasa $\%$ & Ceniza $\%$ \\
\hline T1 & 70,80 & 32,76 & 10,55 & 2,73 \\
T2 & 66,35 & 34,42 & 9,60 & 2,87 \\
T3 & 72,42 & 31,39 & 13,31 & 2,85 \\
T4 & 61,09 & 30,26 & 12,60 & 2,17 \\
T5 & 68,82 & 36,09 & 11,71 & 2,88 \\
T6 & 68,59 & 30,46 & 16,19 & 2,03 \\
T7 & 65,24 & 12,69 & 12,26 & 3,44 \\
T8 & 62,59 & 38,08 & 12,01 & 3,06 \\
T9 & 56,19 & 38,65 & 10,48 & 3,71 \\
\hline
\end{tabular}

Realizando una comparación entre tratamientos y con un $95 \%$ de nivel de confianza para todos los análisis proximal que muestra la tabla 1 se puede afirmar que el factor temperatura y tiempo tienen efecto significativo sobre el porcentaje de humedad, proteína, grasa y ceniza en la carne de cuy frita sometida a los diferentes tratamientos.

La prueba de rangos múltiples nos dice que hay un riesgo del $5 \%$ para decir que cada par de medias es significa-

Tabla 2. Valores de actividad de agua

\begin{tabular}{cc}
\hline Tratamiento & Actividad de agua (Aw) \\
\hline T1 & 0,944 \\
T2 & 0,906 \\
T3 & 0,872 \\
T4 & 0,895 \\
T5 & 0,853 \\
T6 & 0,826 \\
T7 & 0,822 \\
T8 & 0,845 \\
T9 & 0,843 \\
\hline
\end{tabular}

Tabla 4. Valores de cohesividad (dimensional).

\begin{tabular}{cccccc}
\hline $\begin{array}{c}\text { Parámetro } \\
\text { textural }\end{array}$ & Tratamiento & $\begin{array}{c}\text { Valor } \\
\text { medio }\end{array}$ & $\begin{array}{c}\text { Error } \\
\text { estándar }\end{array}$ & $\begin{array}{c}\text { Intervalos de } \\
\text { confianza al 95\% }\end{array}$ \\
\hline $\begin{array}{c}\text { Cohesividad } \\
\text { (adimensional) }\end{array}$ & T1 & $-2,096$ & 0,405 & $-2,947$ & $-1,245$ \\
& T2 & 0,274 & 0,405 & $-0,577$ & 1,126 \\
& T3 & 0,361 & 0,405 & $-0,491$ & 1,212 \\
& T4 & 0,49 & 0,405 & $-0,362$ & 1,341 \\
& T5 & 0,419 & 0,405 & $-0,432$ & 1,27 \\
& T6 & 0,44 & 0,405 & $-0,411$ & 1,291 \\
& T7 & 0,526 & 0,405 & $-0,325$ & 1,378 \\
& T8 & 0,538 & 0,405 & $-0,313$ & 1,389 \\
& T9 & 0,507 & 0,405 & $-0,344$ & 1,358 \\
\hline
\end{tabular}

tivamente diferente para los tratamientos respecto al porcentaje de proteína y grasa mas no existen diferencias entre la media de los tratamientos de humedad y ceniza. Makinson et al. (1987) compararon la absorción de grasa de alimentos de origen vegetal con alimentos de origen animal fritos en abundante grasa a $175^{\circ} \mathrm{C}$, concluyendo que los alimentos de origen vegetal que inicialmente contenían mayor cantidad de agua y bajo contenido de grasa, absorbían más grasa de fritura que los alimentos de origen animal.

\section{Actividad de agua}

La tabla 2 muestra las actividades de agua de las diferentes muestras sometidos a diferentes tratamientos. La prueba de rangos múltiples para los pares de medias de los tratamientos indica que estos pares muestran diferencias estadísticamente significativas con un nivel del $95.0 \%$ de confianza, sin embargo no hay diferencias estadísticas entre la media de los tratamientos T8 y T9.

\section{Determinación Índice de peróxido}

Respecto al índice de peróxido los análisis realizados a las muestras de los diferentes tratamientos no reportaron. El índice de peróxido determina el contenido de hidroperóxidos y ofrece una medida de la oxidación de los ácidos grasos insaturados (Allen et al., 1982; Matissek et al., 1998) a los que fueron sometidas las muestras.

\section{Textura}

Las tablas 3, 4, 5, 6 y 7 muestras los valores de los parámetros texturales de elasticidad, cohesividad, masticabilidad, gomosidad y dureza, tomadas a los tratamientos. Según análisis de varianza con un $95.0 \%$ de nivel de confianza para todos los parámetros texturales, el factor temperatura tienen un efecto estadísticamente significativo sobre elasticidad, la temperatura y tiempo de fritura tienen un

Tabla 3. Valores de elasticidad ( $\mathrm{mm})$

\begin{tabular}{cccccc}
\hline $\begin{array}{c}\text { Parámetro } \\
\text { textural }\end{array}$ & Tratamiento & $\begin{array}{c}\text { Valor } \\
\text { medio }\end{array}$ & $\begin{array}{c}\text { Error } \\
\text { estándar }\end{array}$ & \multicolumn{2}{c}{$\begin{array}{c}\text { Intervalos de } \\
\text { confianza al 95 \% }\end{array}$} \\
\hline Elasticidad & T1 & 5,453 & 0,363 & 4,69 & 6,217 \\
$(\mathrm{~mm})$ & $\mathrm{T} 2$ & 5,083 & 0,363 & 4,32 & 5,847 \\
& $\mathrm{~T} 3$ & 4,977 & 0,363 & 4,213 & 5,74 \\
& $\mathrm{~T} 4$ & 2,687 & 0,363 & 1,923 & 3,45 \\
& $\mathrm{~T} 5$ & 3,024 & 0,363 & 2,261 & 3,787 \\
& $\mathrm{~T} 6$ & 3,363 & 0,363 & 2,6 & 4,127 \\
& $\mathrm{~T} 7$ & 4,523 & 0,363 & 3,76 & 5,287 \\
& $\mathrm{~T} 8$ & 5,34 & 0,363 & 4,577 & 6,103 \\
& $\mathrm{~T} 9$ & 5,287 & 0,363 & 4,523 & 6,05 \\
\hline
\end{tabular}

Tabla 5. Valores de masticabilidad (g/mm).

\begin{tabular}{lccccc}
\hline $\begin{array}{c}\text { Parámetro } \\
\text { textural }\end{array}$ & Tratamiento & $\begin{array}{c}\text { Valor } \\
\text { medio }\end{array}$ & $\begin{array}{c}\text { Error } \\
\text { estándar }\end{array}$ & $\begin{array}{c}\text { Intervalos de } \\
\text { confianza al 95\% }\end{array}$ \\
\hline $\begin{array}{l}\text { Masticabilidad } \\
(\mathrm{g} / \mathrm{mm})\end{array}$ & T1 & -100289 & 25436,5 & -153729 & $-46848,7$ \\
& T2 & 16837 & 25436,5 & $-36603,3$ & 70277,2 \\
& T3 & 28269,9 & 25436,5 & $-25170,3$ & 81710,2 \\
& T4 & 1944,96 & 25436,5 & $-51495,3$ & 55385,2 \\
& T5 & 2680,75 & 25436,5 & $-50759,5$ & 56121 \\
& T6 & 3281,18 & 25436,5 & $-50159,1$ & 56721,4 \\
& T7 & 5892,72 & 25436,5 & $-47547,5$ & 59333 \\
& T8 & 7382,92 & 25436,5 & $-46057,3$ & 60823,2 \\
& T9 & 11329,1 & 25436,5 & $-42111,1$ & 64769,4 \\
\hline
\end{tabular}


Toro, G. et al. Influencia del tiempo y temperatura de fritura en la textura y perfil lipidico en carne de cuy (cavia porcellus) frita.

Tabla 6. Valores de gomosidad (g).

\begin{tabular}{cccccc}
\hline $\begin{array}{c}\text { Parámetro Tratamiento } \\
\text { textural }\end{array}$ & $\begin{array}{c}\text { Valor } \\
\text { medio }\end{array}$ & $\begin{array}{c}\text { Error } \\
\text { estandar }\end{array}$ & \multicolumn{2}{c}{$\begin{array}{c}\text { Intervalos de } \\
\text { confianza al 95\% }\end{array}$} \\
\hline $\begin{array}{c}\text { Gomosidad } \\
\text { (g) }\end{array}$ & T1 & -18307 & 4404,44 & $-27560,4$ & $-9053,6$ \\
& T2 & 3233,65 & 4404,44 & $-6019,75$ & 12487,1 \\
& T3 & 5436,54 & 4404,44 & $-3816,87$ & 14689,9 \\
& T4 & 731,94 & 4404,44 & $-8521,46$ & 9985,34 \\
& T5 & 871,606 & 4404,44 & $-8381,8$ & 10125 \\
& T6 & 857,956 & 4404,44 & $-8395,45$ & 10111,4 \\
& T7 & 1283,3 & 4404,44 & $-7970,11$ & 10536,7 \\
& T8 & 1448,68 & 4404,44 & $-7804,73$ & 10702,1 \\
& T9 & 2165,14 & 4404,44 & $-7088,27$ & 11418,5 \\
\hline
\end{tabular}

Tabla 7. Valores de dureza $(\mathrm{g})$.

\begin{tabular}{cccccc}
\hline $\begin{array}{c}\text { Parámetro } \\
\text { textural }\end{array}$ & Tratamiento & $\begin{array}{c}\text { Valor } \\
\text { medio }\end{array}$ & $\begin{array}{c}\text { Error } \\
\text { estándar }\end{array}$ & $\begin{array}{c}\text { Intervalos de } \\
\text { confianza al 95\% }\end{array}$ \\
\hline Dureza (g) & T1 & 13691 & 3132.5 & 7109.85 & 20272.2 \\
& T2 & 15082.7 & 3132.5 & 8501.52 & 21663.8 \\
& T3 & 15254.3 & 3132.5 & 8673.18 & 21835.5 \\
& T4 & 1487,2 & 3132.5 & -5093.95 & 8068.35 \\
& T5 & 2330,17 & 3132.5 & -4250.98 & 8911.32 \\
& T6 & 1901,8 & 3132.5 & -4679.35 & 8482.95 \\
& T7 & 2375,33 & 3132.5 & -4205.82 & 8956.48 \\
& T8 & 2708 & 3132.5 & -3873.15 & 9289.15 \\
& T9 & 4635.67 & 3132.5 & -1945.48 & 11216.8 \\
\hline
\end{tabular}

Tabla 8. Valores de perfil de ácido graso $(\mathrm{mg} / 100 \mathrm{~g}$ de muestra).

\begin{tabular}{|c|c|c|c|c|c|c|c|c|c|c|c|}
\hline \multirow[t]{2}{*}{$\mathrm{N}^{\circ}$} & \multirow[t]{2}{*}{ Acido Graso } & \multicolumn{10}{|c|}{ Ácidos Grasos mg / $100 \mathrm{~g}$ MUESTRAS } \\
\hline & & $\mathrm{T} 1$ & T2 & T3 & $\mathrm{T} 4$ & T5 & T6 & T7 & T8 & T9 & MO \\
\hline 1 & Pentadecanoic Acid ME (C15:0) & 70 & 14 & 15 & 16 & 8 & 26 & 18 & 33 & 25 & 5 \\
\hline 2 & Palmitic Acid ME (C 16:0) & 1901 & 561 & 653 & 486 & 282 & 966 & 688 & 809 & 749 & 226 \\
\hline 3 & Palmitoleic Acid ME (C16:1 n9c) & 179 & 0 & 33 & 0 & 0 & 48 & 14 & 28 & 28 & 0 \\
\hline 4 & Heptadecanoic Acid ME (C17:0) & 99 & 42 & 38 & 36 & 19 & 52 & 50 & 79 & 48 & 8 \\
\hline 5 & Stearic Acid ME(C18:0) & 813 & 572 & 506 & 352 & 246 & 511 & 577 & 639 & 476 & 68 \\
\hline 6 & Elaidic Acid ME(C18:1n9t) & 1931 & 258 & 547 & 346 & 154 & 825 & 554 & 580 & 718 & 254 \\
\hline 7 & Oleic Actd ME(C18:1n9c) & 78 & 63 & 55 & 41 & 26 & 54 & 0 & 68 & 69 & 0 \\
\hline 8 & Linoleic Acid ME(C18:2n6c) & 3154 & 1191 & 1222 & 1048 & 612 & 2087 & 1742 & 1606 & 1971 & 498 \\
\hline 9 & Linolenic Acid ME(C18:3n3) & 2739 & 343 & 590 & 509 & 192 & 1668 & 587 & 920 & 928 & 241 \\
\hline
\end{tabular}

MO: muestra carne fresca de cuy.

efecto estadísticamente significativo sobre cohesividad.

La temperatura y tiempo de fritura ni la interacción de estos factores tienen un efecto estadísticamente significativo sobre masticabilidad, ninguno de los factores de temperatura o tiempo y sus interacciones tienen un efecto estadísticamente significativo sobre la Gomosidad y finalmente el factor temperatura tiene un efecto estadísticamente significativo sobre la dureza.

Con respecto a la temperatura, no afecta la dureza y adhesividad, elasticidad, gomosidad y masticabilidad, debido a que éste factor se relaciona directamente con el tiempo de freído y juntos controlan las transformaciones y las características del producto final (Vitrac et al., 2002). Sin embargo, el presente estudio el factor temperatura ni su interacción afectan a la masticabilidad y cohesividad, pero si sobre la elasticidad, cohesividad y dureza.

\section{Ácidos grasos}

La tabla 8 muestra los valores de ácidos grasos en $\mathrm{mg} / 100 \mathrm{~g}$ de muestra, nos indica la presencia de estos y su variabilidad respecto a los tratamientos, mostrándose la presencia de 9 ácidos grasos, y un aumento de todos ellos en comparación con la muestra fresca, resalta la presencia y aumento del ácido linolénico, linoleico y oleico que son omegas 3, 6 y 9 respectivamente. El perfil de ácidos grasos del medio de fritura puede ser modificado debido básicamente a dos posibilidades (Al-Kahtani, 1991): al intercambio graso entre medio de fritura y producto frito; y a la oxidación del sustrato.

\section{CONCLUSIONES}

- Debido a la temperatura y tiempo que necesita el proceso de fritura el contenido de agua está relacionado con la absorción de grasa en la carne de cuy frita.

- Los nutrientes se ve favorecida, principalmente con relación a la retención de la proteína.

- El perfil de textura se ve influido por la temperatura y tiempo de fritura en lo que respecta a cohesividad.

- La aplicación de este método conlleva a un aumento en el contenido de la grasa debido al aceite utilizado para la fritura que influye en el perfil de ácidos grasos.

- Se evidencia un aumento de ácidos grasos omega 3, 6 y 9.

\section{REFERENCIAS BIBLIOGRÁFICAS}

Al-Kahtani.-J. Am. (1991). Estudio de calidad de aceites de fritura usados de restaurantes. (E).-H.A. Oil Chemists'Soc. 68: 857-862.

Allen, R. R., Formo, M. W., Krishnamurthy, R.G., McDermott, G.N., Norris, F. A. and Sonntag, N. O.V. (1982). Physical properties of fats and fatty acids. In Bailey's industrial oil and fat products. (pp. 666-679). New York, USA: John Wiley \& Sons.

Gross, R., Koch, F., Malaga, I., Miranda, A., Schoeneberger, H., and Trugo, L. (1989). Chemical composition and protein quality of some local Andean food sources. Food Chem. 34, 25-34.

Gutiérrez H. y De la Vara R. (2003). Análisis y diseño de experi- 
Toro, G. et al. Influencia del tiempo y temperatura de fritura en la textura y perfil lipídico en carne de cuy (cavia porcellus) frita.

mentos. México: Ed. Mc Graw Hill Interamericana. 571 pp.

Hleap, J. y V. Velasco. (2010). Análisis de las propiedades de textura durante el almacenamiento de salchichas elaboradas a partir de tilapia roja (Oreochromis sp.). Facultad de Ciencias Agropecuarias 8(2): 46-56.

Kita, A., G. Lisińska y G. Gołubowska. (2007). The effects of oils and frying temperatures on the texture and fat content of potato crisps. Food Chemistry 102: 1-5.

Kuehl, R. (2001). Diseño de Experimentos: Principios Estadísticos para el Diseño y Análisis de Investigaciones. 2a. ed. México, D.F. Thomson - Learning. 666 p.

Makinson J.H., Greenfield, M.L., Wong, M.L., Wills, R.B.H. (1987). Fat uptake during deep-fat frying of coated and uncoated foods. J Food Comps Anal. 1:93-101.

Matissek, R., Schnepel, F. M. y Steiner, G. (1998). Análisis de los alimentos: fundamentos, métodos, aplicaciones. Zaragoza, España: Editorial Acribia, S. A. pp. 297-302.

Montgomery, D. (2002). Diseño de análisis de experimentos 2 da Edición. Editorial Limusa Wiley.

Pedredo, D y Pangborn, R. (1996). Evaluación sensorial de alimentos, Métodos analiticos. México D.F.: Editorial Alhambra Mexicana.
Pérez, A. (1997). Consumo de grasas crudas y fritas y su utilización nutritiva de minerales (Tesis doctoral). Universidad Complutense de Madrid, España.

Sanz, T., Primo-Martín, C. y Van Vliet, T. (2007). Characterization of crispness of French fries by fracture and acoustic measurements, effect of pre-frying and final frying times. Food Research International 40:63-70.

Taiwo, K. y O. Baik. (2007). Effects of pre-treatments on the shrinkage and textural properties of fried sweet potatoes. $L W T$ 40: 661-668.

Tavera-Quiroz, M.J., Urriza, M., Pinotti, A. y Bertola, N. (2012). Plasticized methylcellulose coating for reducing oil uptake in potato chips. Journal of the Science of Food and Agriculture, 92 (7) 1346-1353.

Vitrac, O., Dufour, D., Trystram, G. y Raoult-Wack, A. (2002). Characterization of heat and mass transfer during deepfat frying and its effect on cassava chip quality. Journal of Food Engineering 53: 161-176.

Yague, A. (2003). Estudio de utilización de aceites para fritura en establecimientos alimentarios de comidas preparadas. Escuela de prevención y seguridad integral. Bellaterra.

\section{Correspondencia:}

Gina Genoveva Toro Rodríguez: gige0168@hotmail.com
Fecha de Recepción: 04/05/2015

Fecha de Aceptación: 12/11/2015 